\title{
First statement on preparation for the COVID-19 pandemic in large German Speaking University-based radiation oncology departments
}

\author{
Stephanie E. Combs ${ }^{1,2,3^{*}}$, Claus Belka ${ }^{3,4}$, Maximilian Niyazi ${ }^{3,4}$, Stefanie Corradini ${ }^{4}$, Steffi Pigorsch ${ }^{1,2,3}$, Jan Wilkens ${ }^{1}$, \\ Anca L. Grosu ${ }^{5,6}$, Matthias Guckenberger ${ }^{7}$, Ute Ganswindt ${ }^{8}$ and Denise Bernhardt ${ }^{1,2,3}$
}

\begin{abstract}
The COVID-19 pandemic is challenging modern radiation oncology. At University Hospitals, we have a mandate to offer high-end treatments to all cancer patients. However, in times of crisis we must learn to prioritize resources, especially personnel. Compromising oncological outcome will blur all statistics, therefore all measures must be taken with great caution. Communication with our neighboring countries, within societies and between departments can help meet the challenge. Here, we report on our learning system and preparation measures to effectively tackle the COVID-19 challenge in University-Based Radiation Oncology Departments.
\end{abstract}

Keywords: COVID-19, Fractionation, Tandem-teams, Pandemic, Radiotherapy

\section{Introduction}

There is a nationwide concern about the coronavirus disease 2019 (COVID-19) and immediate medical emergency caused by the infection with severe acute respiratory syndrome coronavirus 2 (SARS-CoV-2); currently, this virus is the largest global public health threat leading to a major challenge of medical systems in all countries. The family of Coronaviridae consists of a group of large, single, plus-stranded RNA-viruses which have been isolated from several species; common symptoms in humans include common cold and diarrheal illness [1]. Almost 17 years ago, in China, a new coronavirus termed Severe Acute Respiratory Syndrome Coronavirus (SARS-CoV) caused the SARS outbreak and, within

\footnotetext{
* Correspondence: Stephanie.Combs@tum.de

'Department of Radiation Oncology, Technical University of Munich (TUM), Klinikum rechts der Isar, Ismaninger Straße 22, D-81675 München, Munich, Germany

${ }^{2}$ Department of Radiation Sciences (DRS), Institute of Radiation Medicine (IRM), Ingolstädter Landstraße 1, Neuherberg, Germany

Full list of author information is available at the end of the article
}

weeks, spread to more than a dozen countries on several continents including Asia, Europe, North and South America. The crisis affected over 8000 humans and lead to around 800 deaths [2].

As early as 2015, an international research group from USA, Switzerland, and China reported on the pathogenic potential of SARS-like Coronaviruses (CoVs) which were at that time circulating in Chinese horseshoe bat populations [3]; the group extensively described that these viruses can replicate effectively in primary human airway cells, and demonstrated the potential to affect the mouse lung and lead to considerable pathogenesis. Alarmingly, their experiments showed that available SARS-based therapeutics, including immunotherapeutics, vaccines, antibodies and other prophylactic modalities failed to neutralize and protect from CoVs. While elucidating the pathogenic potential, the authors warned of crossspecies transmission.

Only 5 years later, this virus has become a lethal treat for humans; in December of 2019, Wuhan was the 
starting point of a worldwide pandemic, which supposedly originated from Wuhan's wild animal market [4-6]. At the end of January 2020, the first case of a positively tested patient was reported in Germany in Munich, Bavaria, and according to the current status as of March 22, 2020, 18,610 cases were reported in Germany, including 55 deaths (https://www.rki.de/DE/ Content/InfAZ/N/Neuartiges_Coronavirus/Fallzahlen. html).

Radiation Oncology (RO) is a key discipline in oncology and currently more than $50-60 \%$ of all cancer patients are treated with radiotherapy at some point of their disease [7]. Most curative treatment regimens include radiotherapy, many sequentially with surgery, chemotherapy, or as concomitant treatments. Albeit the fear of COVID-19 is currently crossfading all other illnesses, it is the mission of radiation oncologists and other cancer care physicians to courageously take sides for our cancer patients.

We are now faced with increasing hygiene measures, more complicated and difficult treatment procedures aggravated by facial masks and personal protection equipment [8]. The number of employee illnesses is increasing and jeopardizing our efforts to secure continuous patient treatments. While most university-based radiation oncology departments are equipped with highend radiotherapy devices which offer innovative radiation treatments on a high and evidence-based level, human resources are the most precious asset in the COVID-19 challenge.

Over the last years, independently of any pandemic scenario, there has been a wave of arguments that foster hypofractionated treatments in radiation oncology. Most societies actively supporting those concepts are clearly driven by a force of limited treatment capacity paired with a constricted medical system; this forefront is most actively driven by physicians in the United Kingdom (UK). Others have followed, and in indications such as breast cancer [9] and, now following, prostate cancer, concepts of hypofractionation are spreading [10]. While certain low-risk indications might not be undertreated, there is a quiet word of caution for high-risk, fast growing tumors and other factors or tumor biology and normal tissue, that most likely will lead to undertreatment with hypofractionated regimens [11]. In metastatic disease, the argument for hypofractionation is clearly driven by the reduced survival times of palliative patients; however, lessons learned from the treatment of vertebral metastases have nicely shown that short hypofractionated treatments such as $5 \times 4$ Gy or $1 \times 8$ Gy for vertebral metastases may help for short term pain reduction, but longer-term local control is significantly lower than with 10-13 × $3 \mathrm{~Gy}$, and even higher with 40Gy in 2 Gy fractions [12-18]. Estimating life expectancy is always controversial, even among advanced health care providers and with the help of high-end diagnostic, molecular markers and prognostic scores, [19-25].

Now, in our world of high-end radiation oncology service, we are facing a new challenge: keeping our radiation oncology service up and running during the COVID-19 challenge. In spite of specialized and enforced hygiene measures, the crisis has triggered every unit to activate and improve emergency scenarios. Considering potential limitations of personnel capacity, all emergency measures will include, at a certain point, hypofractionated and very pragmatic fractionation schedules. These should ideally be evidence based. However, all measures must be associated with the appropriate word of caution not to compromise oncological outcome if not ultimately necessary due to collapsing resources.

Preparing our radiation oncology departments should therefore follow a series of regulations and measures, to ensure high-end oncological treatment as long as possible.

Overall, personell, patient and device hygiene are the most important measure on wards, radiotherapy units, chemotherapy treatment rooms, administration areas, offices as well as all other public areas. Adequate disinfectants must be provided, at the entrance to the hospital to the ward, to the radiotherapy unit etc., and patients as well as staff must be educated on the effective use of all disinfectants used. For superficial disinfectants, it is important to use quickly active solutions so the radiotherapy scheduling does not come to a standstill. One critical issue in this special pandemic situation is the availability of protective clothing. As long as sufficient numbers of protective gear are available, surgical masks should be worn according to the indications continuously updated by the World Health Organization (WHO) [26], the Robert Koch Institute (RKI) [27], as well as the respective hospital standard operating procedures (SOP). Staff and patients must be advised that masking protects both sides. The lesson of not protecting the medical staff from the very beginning can be drawn from the dramatic Italian experience. The medical staff has become not only a casualty itself, but also a potential source of infection that is transmitted to every patient they meet and treat $[28,29]$. In cases of SARSCoV-2 positivity, a more advanced personal protection equipment is needed and includes: disposable overalls (tunics and/or trousers), disposable gowns and eye protection should be used. FFP2 masks and overshoes are not recommended in all institutions and countries. Visits of relatives and accompanying people should be stopped as early as possible to departments dealing with cancer patients [30]. 
- If the resources allow it, cancer patients, especially those undergoing chemotherapy or those with immunosuppression should wear adequate protective masks. A recent analysis from Wuhan, published in Lancet Oncology revealed that patients with cancer might have a higher risk of COVID-19 than individuals without cancer. Additionally, the study showed that patients with cancer had poorer outcomes and more rapid deterioration from COVID-19 [31, 32]. The authors even concluded, that chemotherapy should be postponed if possible.

- In hospitals where there is no central triage unit, it is recommended to carry out a triage at the entrance of the radiotherapy facilities for the verification of symptomatic patients or the evaluation of contacts to SARS-CoV-2 positive patients in everyone accessing the radiotherapy areas. Standardized questionnaires and measuring the body temperature are advisable. Furthermore, outpatients may be called by telephone 1 day in advance to their appointments to screen for symptoms and ask for contacts to SARS-CoV-2 positive patients/recent stays in so called "risk areas".

- Firstly, the management team of the Department of Radiation Oncology must be clearly identified and the executive power of each member of the management team must be clarified and communicated to the entire team in this special situation. Secondly, the management team must be divided into a tandem operation team (ideally $50 \%$ on site, $50 \%$ off site) to allow for backup solutions. Through this measure it can be secured that the executive force of the Department is not compromised throughout the crisis. The management team should communicate via teleconferencing systems on a regular basis to communicate all details and exchange any changes of situation and not meet in person.

- The staff of the Department of Radiation Oncology outside of areas ultimately relevant for the retention of clinical department operations should be limited. The offices, research areas, wards as well as other clinical areas of the department should be restricted to people working in system-critical areas. The personnel may include staff for the basic operations of the Department, including, in particular, indispensable employees in administration and patient care. For research areas, specific institutional guidelines can be released. In some centers, critical persons include personnel involved in animal husbandry and animal research facilities, as well as the supervisors of scientific long-term experiments and technical infrastructure. In other scenarios, research animal facilities are generally closed and all staff is moved towards maintaining clinical infrastructure and operations, as needed. The management team should strictly supervise and coordinate these measures and enable home-office possibilities.

- For all system-relevant areas, a tandem-staffing must be planned at the earliest possible timepoint. Tandems comprise two different persons who work as back up for each other. They are not supposed to be in physical contact, they are not allowed to enter the same room at the same time. Importantly, communication between tandems has to be ensured at all time by digital means to maintain tandem members at the same level of information. These tandem teams should be built especially for doctors, with special focus on the group of radiation protection physicians to comply with regulatory responsibilities at any timepoint of the crisis. Also, technicians, medical physics experts (MPEs), radiation safety officers as well as secretaries and nurses should be places in tandem teams who stand in continuous mutual consultation. These tandem groups regularly switch on- and off-site assignments every fortnight to overcome the 14-day half-life of the SARS-CoV-2. Theses tandem groups depend on the size and resources of the department, however, even subgroup tandem-staffing could be a compromise solution.

- Some institutions recommend that all other staff who is not working in system-critical areas should work in home office, if possible. Special assignments for the off-site work will be distributed by the management team, the group leaders or other supervisors. All off-site workers must be available to supervisors as well as colleagues via landline, mobile phone or e-mail. However, if this is not possible and on-site working is performed, social distancing and special hygiene requirements are essential. Digital communication should be evaluated were possible.

- In order to meet the special challenges of these days, management and the works council of some institutions have reached an agreement on the subject of "confidential working hours", which makes the current situation easier for both employers and employees. This means, the employer is not actively checking working hours, and at some institutions electronic time recording is de-activated since many individuals will be working off-site or in home office situations.

- All planned leave days of personnel essential for clinical operations should be cancelled during the team of crisis. This ensures that essential workforce is present and can be recruited to the department as necessary. Another option is to remain on stand-by, for immediate deployment when other personnel is 
absent. In all other groups of the workforce not essential for clinical operations staff may be asked to take all vacation days possible during this time.

- Conferences and interdisciplinary tumor boards should be switched to digital solutions like email and video conferences. If this is not possible, only relevant staff with executive functions or experts should participate to minimize group size and exposure in order to prevent viral spread between staff and different departments.

- Interdisciplinary case discussions (tumorboards) are a standard in oncology. In these special times, these discussions should not be compromised. Rather, these conferences should be strengthened and intensified to find the optimal solution for each patient together with the neighboring disciplines, such as hematology/oncology and surgery.

- All follow-ups should be critically evaluated and postponed if not regarded essential. Outpatient clinics are advised to use online, digital or medical counselling by phone. However, it is important to secure appropriate identification and treatment of critical cases. For all outpatient clinics as well as necessary follow-ups, entry point screening is necessary; patients should fill out a hygiene risk questionnaire asking for possible symptoms and potential residency or travel history to risk areas.

- In order to minimize the risk for both the patients and health care staffing by the repetitive risk of exposure, the indications for radiotherapy must be strictly defined. Table 1 gives an evidence-based overview of possible hypofractionated regimens or observational strategies for a variety of entities which can be considered during this crisis. The potential benefits and risks of altered fractionations should be carefully discussed with the patient. Procrastinating certain pathologies by evaluating the risk/benefit ratio in each individual case is advisable. Moreover, benign diseases should not be treated at all. Importantly, this table does not represent standard-of-care regimens and the fractionations should not be used routinely outside of special crisis situations.

- In times were surgical and anesthesiological capacity might become even more rare, the equieffectivity of radiotherapy and radiochemotherapy regimens as non-invasive treatments should be discussed. For selected indications, literature is summarized in Table 2, 3 and 4.

- Prepare staff and patients how to best mitigate the impact of a 2- to 3-week interruption in treatment. From the aftermaths from hurricane Maria in Puerto Rico there are rough ASTRO guidelines based on the limited evidence available [73-76].
- If possible members of the staff at high risk for severe COVID-19 courses of disease should be identified and, if possible from an organizational point of view, allotted to off-site assignments to allow for optimal social distancing and staff protection. This may include older employees, those with comorbidities or other factors.

As long as possible, the provision of high-end oncological care should be maintained. The despair of several Italian and Spanish Radiation Oncology Departments in the emerging COVID-19 crisis argued for triage and extensive application of hypofractionated and ultimately ultra-hypofractionated radiation regimens [8, 77]. Although the overall pandemic situation might force individuals for this strategy, it must be always kept in mind that oncological care should not be compromised if not ultimately needed, especially in the curative setting. Additionally, all statistics of survival will be massively blurred if broad radiation oncology service is restricted and COVID-19 fear and pandemic crisis force us to use minimalistic radiation efforts.

While most recommendations from other radiation oncology societies are in line with the above-mentioned measures during the COVID-19 outbreak, the treatment of COVID-19 positive patients remains the greatest challenge.

- A continuous triage evaluation is needed to detect an early onset of typical symptoms of COVID-19 (fever, cough, sore throat, shortness of breath, fatigue) in patients already receiving treatments at the Radiation Oncology Department. This should immediately be reported to the management team. Adequate testing and reporting to authorities in positive cases is mandatory. RKI criteria 3 and 4 should be applied to separate patients into those with a less well-founded suspicion: acute respiratory symptoms with/without fever and no stay in regions with COVID-19 cases or clinical/radiological viral pneumonia without alternative diagnosis without exposition risk.

- In patients who are already undergoing radiotherapy and are suspected of having typical COVID-19 symptoms, the treatment should be immediately interrupted, and testing results should be awaited.

- In COVID-19 positive patients, who have not started treatment, it is recommended to postpone treatment initiation whenever medically feasible and does not compromise outcome, survival or quality of life (QoL).

- In SARS-CoV-2 positive patients already undergoing radiotherapy, the continuation of treatment can only proceed when specific measures are taken. Each 


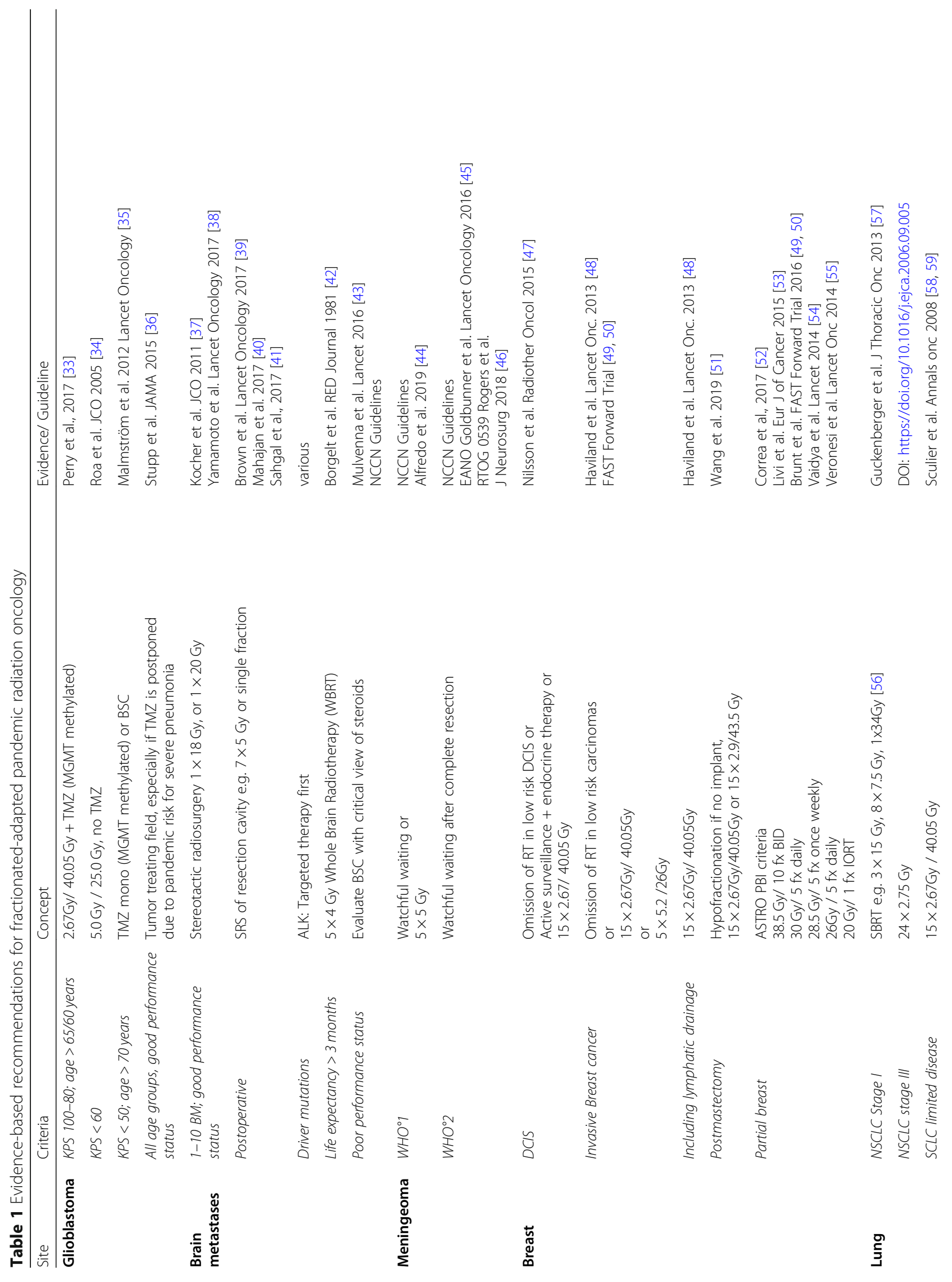




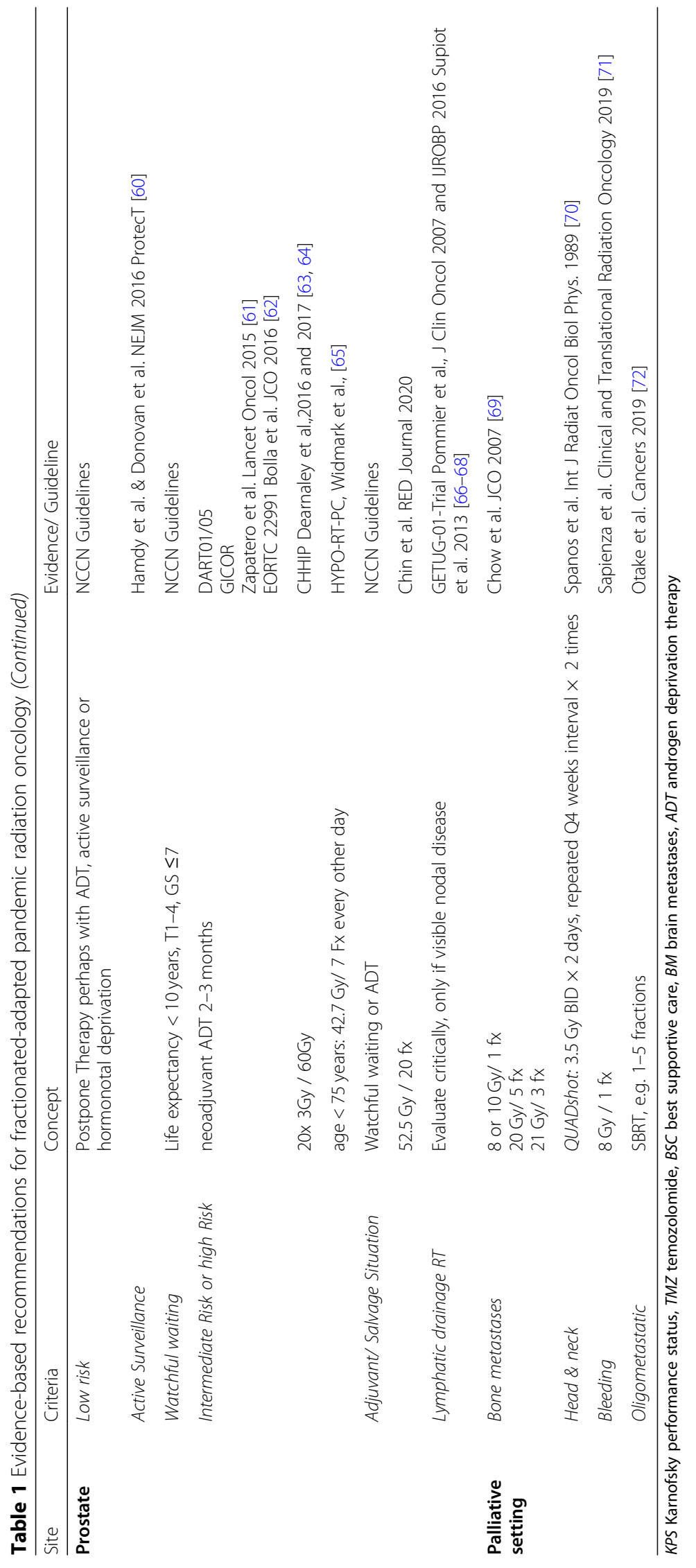




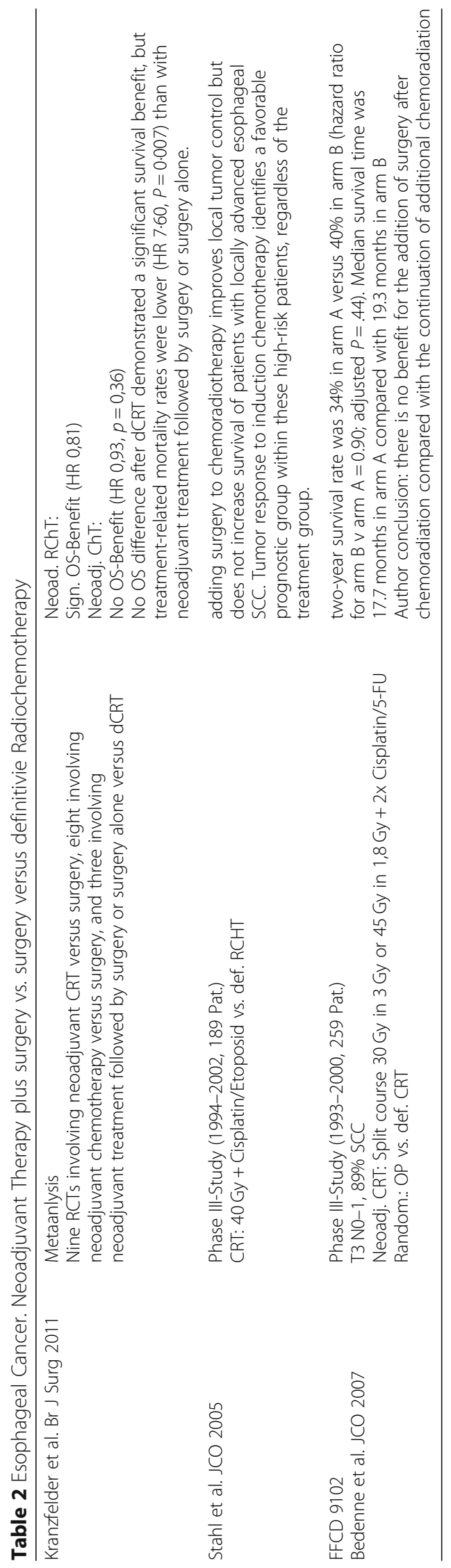




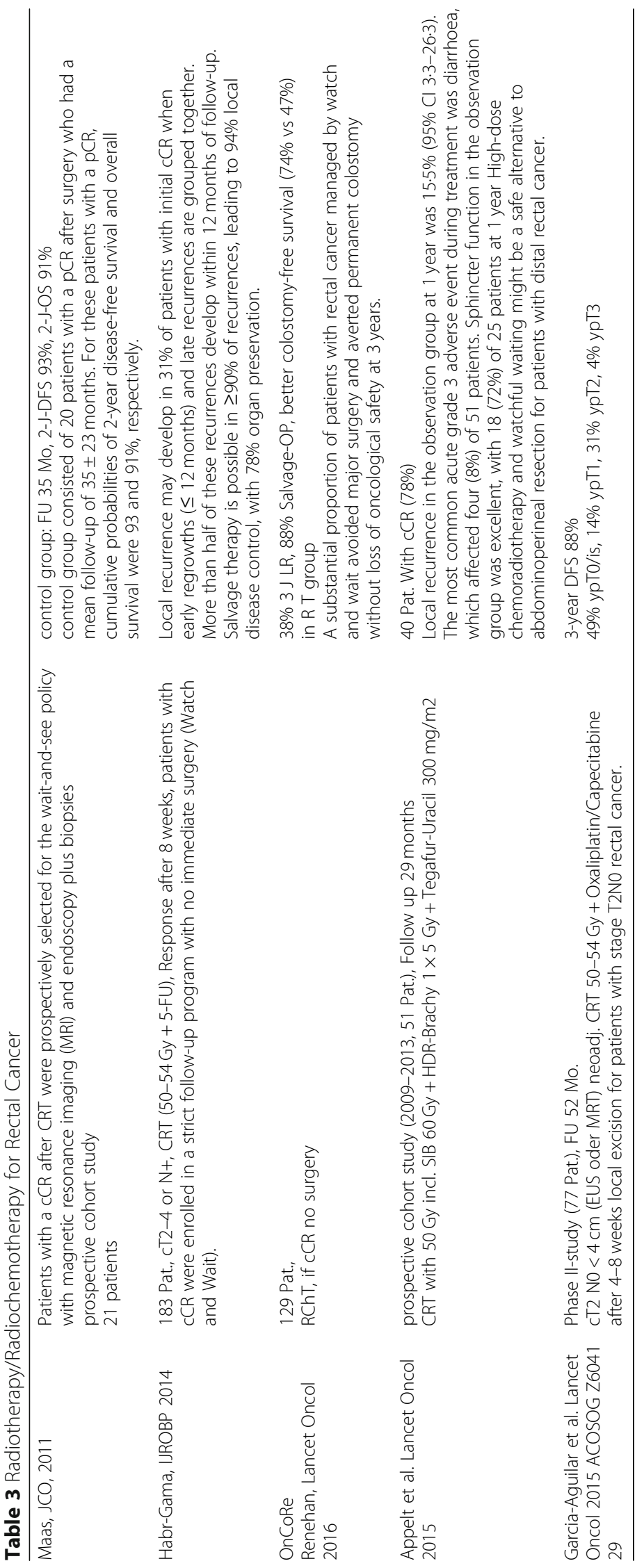




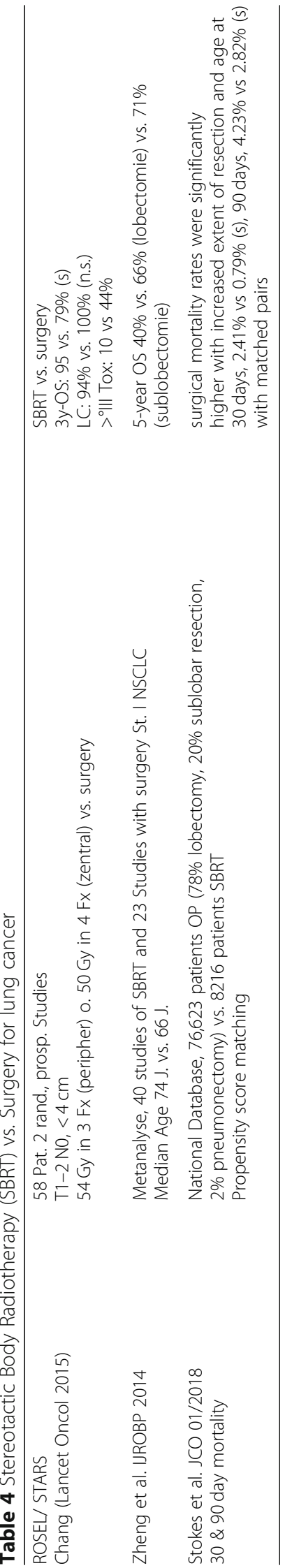


department should individually weigh their resources and evaluated on a case by cases basis (indication for treatment, performance status of the patient, etc.). If these prerequisites cannot be met, a discontinuation of treatment is mandatory. Especially, as the case load of SARS-CoV-2-positive patients is increasing significantly, a strict no-RTpolicy can be decided.

- If treatment continues, these measures must be taken:

oThe treatment has to be carried out under maximal safety conditions to guarantee the protection of health professionals.

oThe patients should be treated at a special linear accelerator, with a specific access route to prevent contact to other patients. This can for example be performed at the end of the regular treatment schedules.

oThe staff needs appropriate personal protection equipment according to institutional guidelines and availability.

oThe equipment and linac must be adequately sanitized at the end of treatment.

- Alternatively, all treatments in SARS-CoV-2 positive patients could be interrupted to avoid infections of the staff and other patients

- In SARS-CoV-2 positive patients declared cured from the disease, a careful evaluation has to be performed prior to proceed radiotherapy with less advanced measures.

Taken together, the way we treat cancer in the coming months will change dramatically during this pandemic. While we are used to counselling patients on their best treatment option, we will be confronted with a whole new dimension - where we will have to balance the benefits of oncological therapies against the increased risk of cancer patients for SARS-CoV-2 infections. The indications and timelines of our radiotherapy treatments may shift, and we might accept higher risks of cancer recurrences over a short-term increase in risk of death from COVID-19. Despite the increased anxiety and uncertainty that all cancer patients face while awaiting time-dependent treatments, this could even be worse during these days of social distancing and unavailable treatment options. Please consider this aspect during your clinical routine. The selected and presented scientific evidence, it's interpretation and translation into radiation oncology specific recommendations by this multi-institutional and international collaboration need to be put into the very special context of the COVID-19 pandemic. Firstly, there is still very little knowledge or even evidence available, yet. Available data is sometimes conflicting and incomplete. This knowledge is luckily expanding at enormous speed, which makes however the generation of a founded synthesis difficult. In such a dynamic situation, this manuscript therefore reflects a snapshot, which may become outdated rapidly. Recommendations aim to maximize cancer care of all patients, best-as possible protection of our health care workers and simultaneously rigid suppression of the pandemic spread. It is obvious that not all three goals can be followed and can be achieved to $100 \%$, compromises in one or the other way will have to be made. Such multifactorial problems will require difficult decisions: decisions will be influenced by various stakeholder (government, health authorities, hospital and university administration), will be restricted by logistical and financial aspects, will need to follow the respective legal frameworks, will need to be put into the political and cultural context, and will at the same time need to consider the individual patient and their families. Consequently, there will be differences between countries, states, institutions and even between individual clinicians, all trying their best in such situations of crisis. Finally, we do not know, yet, whether the structured planning described in this manuscript will stand or survive the potentially dramatic developments in the future, where irrational or hard actions might become reality.

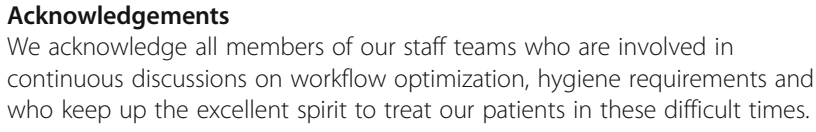

\section{Authors' contributions}

SEC, JW, SP and DB drafted the manuscript. SEC, DB, MN, SC, ALG, CB, UG, FL and $M G$ helped compile the literature and provided essential content to manuscript writing. All co-authors corrected and manuscript and approved the final version.

\section{Funding}

The work was funded by the participating departments. No external funding is related to this manuscript.

\section{Availability of data and materials}

All references are cited in the manuscript. There is no additional data and material involved.

Ethics approval and consent to participate

Ethics approval was not necessary for the comprehensive review article. All co-authors have agreed to participate.

Consent for publication

All co-authors have head and approved the final manuscript.

\section{Competing interests}

The authors declare there are not competing interests.

\section{Author details}

${ }^{1}$ Department of Radiation Oncology, Technical University of Munich (TUM), Klinikum rechts der Isar, Ismaninger Straße 22, D-81675 München, Munich, Germany. ' Department of Radiation Sciences (DRS), Institute of Radiation Medicine (IRM), Ingolstädter Landstraße 1, Neuherberg, Germany. ${ }^{3}$ Deutsches Konsortium für Translationale Krebsforschung (DKTK), Partner Site Munich, Munich, Germany. ${ }^{4}$ Department of Radiation Oncology, University Hospital, LMU, Munich, Germany. ${ }^{5}$ Department of Radiation Oncology, Medical Center, 
University of Freiburg, Freiburg im Breisgau, Germany. ${ }^{6}$ Deutsches Konsortium für Translationale Krebsforschung (DKTK), Partner Site Freiburg, Freiburg im Breisgau, Germany. ${ }^{7}$ Department of Radiation Oncology, University Hospital Zurich, University of Zurich, Zurich, Switzerland. ${ }^{8}$ Department of Radiation Oncology, Medical University of Innsbruck, Innsbruck, Austria.

\section{Received: 25 March 2020 Accepted: 31 March 2020}

\section{Published online: 07 April 2020}

\section{References}

1. Fehr AR, Perlman S. Coronaviruses: an overview of their replication and pathogenesis. Methods Mol Biol. 2015;1282:1-23.

2. Peiris JS, Guan Y, Yuen KY. Severe acute respiratory syndrome. Nat Med. 2004;10(12 Suppl):S88-97.

3. Menachery VD, et al. A SARS-like cluster of circulating bat coronaviruses shows potential for human emergence. Nat Med. 2015;21 (12):1508-13.

4. Rocklov J, Sjodin H, Wilder-Smith A. COVID-19 outbreak on the diamond princess cruise ship: estimating the epidemic potential and effectiveness of public health countermeasures. J Travel Med. 2020.

5. Rothan HA, Byrareddy SN. The epidemiology and pathogenesis of coronavirus disease (COVID-19) outbreak. J Autoimmun. 2020:102433.

6. Adhikari SP, et al. Epidemiology, causes, clinical manifestation and diagnosis, prevention and control of coronavirus disease (COVID-19) during the early outbreak period: a scoping review. Infect Dis Poverty. 2020;9(1):29.

7. Baumann $\mathrm{M}$, et al. Radiation oncology in the era of precision medicine. Nat Rev Cancer. 2016;16(4):234-49.

8. Filippi AR, Russi E, Magrini SM, Corvò R. Covid-19 outbreak in northern Italy: First Practical Indications for Radiotherapy Departments. Int J Radiat Oncol Biol Phys. 2020.

9. Budach W, Bolke E, Matuschek C. Hypofractionated Radiotherapy as Adjuvant Treatment in Early Breast Cancer. A Review and Meta-Analysis of Randomized Controlled Trials. Breast Care (Basel). 2015;10(4):240-5.

10. Hocht $\mathrm{S}$, et al. Hypofractionated radiotherapy for localized prostate cancer Strahlenther Onkol. 2017:193(1):1-12.

11. Eccles SA, et al. Critical research gaps and translational priorities for the successful prevention and treatment of breast cancer. Breast Cancer Res. 2013;15(5):R92.

12. Douglas $\mathrm{S}$, et al. Prognostic factors for different outcomes in patients with metastatic spinal cord compression from cancer of unknown primary. BMC Cancer. 2012;12:261.

13. Douglas S, Schild SE, Rades D. Metastatic spinal cord compression in patients with cancer of unknown primary. Estimating the survival prognosis with a validated score. Strahlenther Onkol. 2012:188(11):1048-51.

14. Douglas S, Schild SE, Rades D. A new score predicting the survival of patients with spinal cord compression from myeloma. BMC Cancer. 2012;12:425

15. Rades $\mathrm{D}$, Abrahm JL. The role of radiotherapy for metastatic epidural spinal cord compression. Nat Rev Clin Oncol. 2010;7(10):590-8.

16. Rades D, Bajrovic A, Bartscht T. Predictive factors and a survival score for patients irradiated for metastatic spinal cord compression from carcinoma of the salivary glands. Anticancer Res. 2017;37(12):7011-5.

17. Rades $D$, et al. Forecasting survival probabilities after radiotherapy of metastatic epidural spinal cord compression from colorectal Cancer in the elderly. Anticancer Res. 2016;36(4):1829-33.

18. Rades $D$, et al. Dose escalation for metastatic spinal cord compression in patients with relatively radioresistant tumors. Int J Radiat Oncol Biol Phys. 2011;80(5):1492-7.

19. Combs SE, et al. Re-irradiation of recurrent gliomas: pooled analysis and validation of an established prognostic score-report of the radiation oncology group (ROG) of the German Cancer consortium (DKTK). Cancer Med. 2018;7(5):1742-9.

20. Niyazi $\mathrm{M}$, et al. Independent validation of a new reirradiation risk score (RRRS) for glioma patients predicting post-recurrence survival: a multicenter DKTK/ROG analysis. Radiother Oncol. 2018;127(1):121-7.

21. Niyazi $\mathrm{M}$, et al. Validation of the prognostic Heidelberg re-irradiation score in an independent mono-institutional patient cohort. Radiat Oncol. 2014;9:128.

22. Kessel KA, Combs SE. Digital biomarkers: importance of patient stratification for re-irradiation of glioma patients - review of latest developments regarding scoring assessment. Phys Med. 2019;67:20-6.
23. Kessel KA, et al. Modification and optimization of an established prognostic score after re-irradiation of recurrent glioma. PLoS One. 2017;12(7):e0180457.

24. Kessel KA, et al. Validation of an established prognostic score after reirradiation of recurrent glioma. Acta Oncol. 2017:56(3):422-6.

25. Rades $\mathrm{D}$, et al. Prognostic factors predicting functional outcomes, recurrence-free survival, and overall survival after radiotherapy for metastatic spinal cord compression in breast cancer patients. Int J Radiat Oncol Biol Phys. 2006;64(1):182-8.

26. World Health Organization (WHO). www. who.int.

27. Robert Koch Institute. https://www.rki.de/DE/Home/homepage_node.html.

28. Li R, et al. Substantial undocumented infection facilitates the rapid dissemination of novel coronavirus (SARS-CoV2). In: Science; 2020.

29. Nacoti, M.C. A; Giupponi A; Brambillasca P; Lussana F; Pisano M; Goisis, G; Bonacina, D; Fazzi, F; Naspro, R; Longhi, L; Cereda, M; Montaguti, C, At the Epicenter of the Covid-19 Pandemic and Humanitarian Crises in Italy: Changing Perspectives on Preparation and Mitigation. N Engl J Med Catalyst, 2020. in press.

30. The First Affiliated Hospital, Z.U.S.o.M. Handbook of COVID-290 Prevention and Treatment. 2020

31. Wang H, Zhang L. Risk of COVID-19 for patients with cancer. Lancet Oncol. 2020.

32. Liang $W$, et al. Cancer patients in SARS-CoV-2 infection: a nationwide analysis in China. Lancet Oncol. 2020;21(3):335-7.

33. Perry JR, Laperriere N, Mason WP. Radiation plus Temozolomide in patients with Glioblastoma. N Engl J Med. 2017;376(22):2197.

34. Roa W, et al. Abbreviated course of radiation therapy in older patients with glioblastoma multiforme: a prospective randomized clinical trial. J Clin Oncol. 2004;22(9):1583-8.

35. Malmstrom A, et al. Temozolomide versus standard 6-week radiotherapy versus hypofractionated radiotherapy in patients older than 60 years with glioblastoma: the Nordic randomised, phase 3 trial. Lancet Oncol. 2012; 13(9):916-26.

36. Stupp R, et al. Maintenance therapy with tumor-treating fields plus Temozolomide vs Temozolomide alone for Glioblastoma: a randomized clinical trial. JAMA. 2015;314(23):2535-43.

37. Kocher $\mathrm{M}$, et al. Adjuvant whole-brain radiotherapy versus observation after radiosurgery or surgical resection of one to three cerebral metastases: results of the EORTC 22952-26001 study. J Clin Oncol. 2011;29(2):134-41.

38. Yamamoto $\mathrm{M}$, et al. Stereotactic radiosurgery for patients with multiple brain metastases (JLGK0901): a multi-institutional prospective observational study. Lancet Oncol. 2014:15(4):387-95.

39. Brown PD, et al. Effect of radiosurgery alone vs radiosurgery with whole brain radiation therapy on cognitive function in patients with 1 to 3 brain metastases: a randomized clinical trial. JAMA. 2016;316(4):401-9.

40. Mahajan A, et al. Post-operative stereotactic radiosurgery versus observation for completely resected brain metastases: a single-Centre, randomised, controlled, phase 3 trial. Lancet Oncol. 2017;18(8):1040-8.

41. Sahgal A, et al. Stereotactic radiosurgery alone for multiple brain metastases? A review of clinical and technical issues. Neuro-Oncology. 2017; 19(suppl_2):ii2-ii15.

42. Borgelt $\mathrm{B}$, et al. Ultra-rapid high dose irradiation schedules for the palliation of brain metastases: final results of the first two studies by the radiation therapy oncology group. Int J Radiat Oncol Biol Phys. 1981;7(12):1633-8.

43. Mulvenna $\mathrm{P}$, et al. Dexamethasone and supportive care with or without whole brain radiotherapy in treating patients with non-small cell lung cancer with brain metastases unsuitable for resection or stereotactic radiotherapy (QUARTZ): results from a phase 3, non-inferiority, randomised trial. Lancet. 2016;388(10055):2004-14.

44. Alfredo C, et al. Normofractionated stereotactic radiotherapy versus CyberKnife-based hypofractionation in skull base meningioma: a German and Italian pooled cohort analysis. Radiat Oncol. 2019;14(1):201.

45. Goldbrunner $R$, et al. EANO guidelines for the diagnosis and treatment of meningiomas. Lancet Oncol. 2016;17(9):e383-91.

46. Rogers $\mathrm{L}$, et al. Intermediate-risk meningioma: initial outcomes from NRG oncology RTOG 0539. J Neurosurg. 2018;129(1):35-47.

47. Nilsson C, Valachis A. The role of boost and hypofractionation as adjuvant radiotherapy in patients with DCIS: a meta-analysis of observational studies. Radiother Oncol. 2015;114(1):50-5.

48. Haviland JS, et al. The UK standardisation of breast radiotherapy (START) trials of radiotherapy hypofractionation for treatment of early breast cancer: 10-year follow-up results of two randomised controlled trials. Lancet Oncol. 2013;14(11):1086-94 
49. Brunt AM, et al. Acute skin toxicity associated with a 1-week schedule of whole breast radiotherapy compared with a standard 3-week regimen delivered in the UK FAST-forward trial. Radiother Oncol. 2016;120(1):114-8.

50. group, F.T, et al. First results of the randomised UK FAST Trial of radiotherapy hypofractionation for treatment of early breast cancer (CRUKE) 04/015). Radiother Oncol. 2011;100(1):93-100.

51. Wang SL, et al. Hypofractionated versus conventional fractionated postmastectomy radiotherapy for patients with high-risk breast cancer: a randomised, non-inferiority, open-label, phase 3 trial. Lancet Oncol. 2019; 20(3):352-60.

52. Correa C, et al. Accelerated partial breast irradiation: executive summary for the update of an ASTRO evidence-based consensus statement. Pract Radiat Oncol. 2017;7(2):73-9.

53. Livi $\mathrm{L}$, et al. Accelerated partial breast irradiation using intensity-modulated radiotherapy versus whole breast irradiation: 5-year survival analysis of a phase 3 randomised controlled trial. Eur J Cancer. 2015;51(4):451-63.

54. Vaidya JS, et al. Risk-adapted targeted intraoperative radiotherapy versus whole-breast radiotherapy for breast cancer: 5 -year results for local control and overall survival from the TARGIT-A randomised trial. Lancet. 2014; 383(9917):603-13.

55. Veronesi $U$, et al. Intraoperative radiotherapy versus external radiotherapy for early breast cancer (ELIOT): a randomised controlled equivalence trial. Lancet Oncol. 2013;14(13):1269-77.

56. Videtic GM, et al. Long-term follow-up on NRG oncology RTOG 0915 (NCCTG N0927): a randomized phase 2 study comparing 2 stereotactic body radiation therapy schedules for medically inoperable patients with stage I peripheral non-small cell lung Cancer. Int J Radiat Oncol Biol Phys. 2019;103(5):1077-84

57. Guckenberger M, et al. Safety and efficacy of stereotactic body radiotherapy for stage 1 non-small-cell lung cancer in routine clinical practice: a patternsof-care and outcome analysis. J Thorac Oncol. 2013;8(8):1050-8.

58. Sculier JP, et al. A phase III randomised study of concomitant induction radiochemotherapy testing two modalities of radiosensitisation by cisplatin (standard versus daily) for limited small-cell lung cancer. Ann Oncol. 2008; 19(10):1691-7.

59. Murray $\mathrm{N}$, et al. Importance of timing for thoracic irradiation in the combined modality treatment of limited-stage small-cell lung cancer. The National Cancer Institute of Canada clinical trials group. J Clin Oncol. 1993; 11(2):336-44.

60. Hamdy FC, et al. 10-year outcomes after monitoring, surgery, or radiotherapy for localized prostate Cancer. N Engl J Med. 2016;375(15): $1415-24$.

61. Zapatero A, et al. High-dose radiotherapy with short-term or long-term androgen deprivation in localised prostate cancer (DART01/05 GICOR): a randomised, controlled, phase 3 trial. Lancet Oncol. 2015;16(3):320-7.

62. Bolla $\mathrm{M}$, et al. Short androgen suppression and radiation dose escalation for intermediate- and high-risk localized prostate Cancer: results of EORTC trial 22991. J Clin Oncol. 2016;34(15):1748-56.

63. Dearnaley D, Hall E. Prostate cancer and hypofractionation: reflections on recent randomised phase III clinical trial results. Transl Androl Urol. 2017;6(1): 134-6.

64. Dearnaley D, et al. Conventional versus hypofractionated high-dose intensity-modulated radiotherapy for prostate cancer: 5-year outcomes of the randomised, non-inferiority, phase 3 CHHiP trial. Lancet Oncol. 2016; 17(8):1047-60.

65. Widmark A, et al. Ultra-hypofractionated versus conventionally fractionated radiotherapy for prostate cancer: 5-year outcomes of the HYPO-RT-PC randomised, non-inferiority, phase 3 trial. Lancet. 2019;394(10196):385-95.

66. Pommier $\mathrm{P}$, et al. Is there a role for pelvic irradiation in localized prostate adenocarcinoma? Update of the long-term survival results of the GETUG-01 randomized study. Int J Radiat Oncol Biol Phys. 2016;96(4):759-69.

67. Pommier $P$, et al. Is there a role for pelvic irradiation in localized prostate adenocarcinoma? Preliminary results of GETUG-01. J Clin Oncol. 2007;25(34): 5366-73.

68. Supiot $\mathrm{S}$, et al. Hypofractionated radiotherapy in prostate cancer. Cancer Radiother. 2013;17(5-6):349-54

69. Chow $\mathrm{E}$, et al. Palliative radiotherapy trials for bone metastases: a systematic review. J Clin Oncol. 2007;25(11):1423-36.

70. Spanos W Jr, et al. Phase II study of multiple daily fractionations in the palliation of advanced pelvic malignancies: preliminary report of RTOG 8502 Int J Radiat Oncol Biol Phys. 1989;17(3):659-61.
71. Sapienza LG, et al. Short-course palliative radiation therapy leads to excellent bleeding control: a single Centre retrospective study. Clin Transl Radiat Oncol. 2019;14:40-6.

72. Otake S, Goto T. Stereotactic Radiotherapy for Oligometastasis. Cancers (Basel). 2019;11(2)

73. Yom SS, Harari PM. When disaster strikes: mitigating the adverse impact on head and neck Cancer patients. Int J Radiat Oncol Biol Phys. 2018;100(4): 838-40.

74. Sandler HM. Role of overall treatment time in the Management of Prostate Cancer Patients: how to manage unscheduled treatment interruptions. Int J Radiat Oncol Biol Phys. 2018;100(4):841-2

75. Roach MC, Robinson CG, Bradley JD. Natural disasters and the importance of minimizing subsequent radiation therapy interruptions for locally advanced lung Cancer. Int J Radiat Oncol Biol Phys. 2018;100(4):836-7.

76. Gay HA, et al. Lessons learned from hurricane Maria in Puerto Rico: practical measures to mitigate the impact of a catastrophic natural disaster on radiation oncology patients. Pract Radiat Oncol. 2019;9(5):305-21.

77. Achard V, et al. Twice- vs. thrice-weekly moderate hypofractionated radiotherapy for prostate cancer: does overall treatment time matter? J Cancer Res Clin Oncol. 2019;145(6):1581-8.

\section{Publisher's Note}

Springer Nature remains neutral with regard to jurisdictional claims in published maps and institutional affiliations.
Ready to submit your research? Choose BMC and benefit from:

- fast, convenient online submission

- thorough peer review by experienced researchers in your field

- rapid publication on acceptance

- support for research data, including large and complex data types

- gold Open Access which fosters wider collaboration and increased citations

- maximum visibility for your research: over $100 \mathrm{M}$ website views per year

At BMC, research is always in progress.

Learn more biomedcentral.com/submissions 